\title{
DEVELOPMENT OF A MESH CLUSTERING ALGORITHM AIMED AT REDUCING THE COMPUTATIONAL EFFORT OF GEARBOXES' CFD SIMULATIONS
}

\author{
MARCO NICOLA MASTRONE \& FRANCO CONCLI \\ Free University of Bolzano, Italy
}

\begin{abstract}
Over the last decade the development of simulation tools led to significant advancements in the design of virtual prototypes. Mechanical design benefitted a lot from this technological progress in terms of costs' reduction. However, in many applications as, for example, gearboxes, the implementation of complex CFD models that involve multiphase simulations with dynamic meshes and turbulence modelling is still a concern in terms of computational time. The main issue is related to the management of the topological modifications of the computational domain during the boundary rotation. For this reason, it is fundamental to have at disposal accurate and time efficient solutions for the correct evaluation of the efficiency and lubrication of geared transmissions already in the early stages of the design. In this work, an automatic algorithm for the reduction of the computational effort associated with the mesh generation and handling in the simulation of gearboxes is presented. The algorithm is based on the creation of a limited number of meshes for the description of the whole wheels' rotation by proper setting the time libraries taking advantage of the cyclicality of the gears' teeth position. Several operating conditions were simulated exploiting this methodology showing a drastic reduction of the simulations' time, making this tool ideal in the investigation of gears' lubrication and efficiency in a limited amount of time. The methodology was implemented in the open-source software OpenFOAM $^{\circledR}$.

Keywords: CFD, mesh handling, lubrication, efficiency, power losses, OpenFOAM ${ }^{\circledR}$, gears.
\end{abstract}

\section{INTRODUCTION}

The technological advancements in computer science that have characterized the last decade offered new possibilities to approach mechanical design. Simulation codes are nowadays widespread both in industry and academia. The reduction of time to market enabled by virtual prototypes as well as the opportunity to investigate operating conditions for which data acquisition can be complex and costly are the fundamental reasons behind such diffusion. These tools are referred to as Computer-Aided Engineering (CAE) among which the most used are Finite Element Analysis (FEA), Multibody Dynamics (MBD) and Computational Fluid Dynamics (CFD). The application of these tools allowed engineers to support experimental findings with numerical results and to analyse (numerically) the behavior of the considered system in several operating conditions. One of the aspects that has benefitted a lot from simulation software packages is related to the study of lubrication and efficiency of geared transmissions. In fact, the application of numerical codes to gearboxes allows one to obtain information on the lubricant distribution and the power dissipation that are usually hard to quantify analytically or to measure experimentally (difficult optical access, need for niche equipment and simplified prototypes, etc.). For these reasons, CFD studies can be beneficial to bridge the gap leading to a deeper understanding of the physical phenomena and to tackle the more and more stringent requirements of energy efficient solutions. One of the main limitations in the simulations of gears is related to the high computational resources needed to handle the topological changes of the domain. In fact, while the gears rotate, the mesh loses its validity and must be updated with a new grid having sufficient quality to ensure the numerical convergence. It is common to need hundreds of meshes to accomplish the 
gears' rotation and to reach the solution convergence. The massive need of valid grids has a severe impact on the computational time.

While it is already possible to efficiently simulate spur gears exploiting extrusion algorithms which reduce the problem to a $2-2.5 \mathrm{D}$ remeshing process [1]-[11], more complex geometries, as helical and bevel gears, cannot benefit from this solution, and tetrahedral meshes must be employed. These geometries have been simulated mainly by exploiting commercial codes requiring massive parallelization and high computational resources both with mesh [12]-[17] and meshless methods [18]-[20], while no work was found in the literature based on opensource software. The authors maintain that a general algorithm applicable for any system configuration can represent an important step in the simulation of gearboxes and in the reduction of computational resources needed for the meshes handling of the domain. In this work, an algorithm that requires a limited number of grids is implemented in the open-source software OpenFOAM ${ }^{\circledR}[21]$ showing a drastic reduction of the computational costs associated with the mesh generation opening new scenarios for a massive introduction of CFD applied to lubrication also in industry.

\section{MATERIALS AND METHODS}

\subsection{Meshing strategy}

To numerically discretize gearboxes, it is fundamental to use a robust mesh generator. Native applications of OpenFOAM ${ }^{\circledR}$ are blockMesh and snappyHexMesh. The former is a utility that builds full a structured mesh providing the user with a perfect control on every aspect of the mesh generation process. The main drawback of this tool is that it is not suitable for gearboxes due to the complex shape of the computational domain which would require very complex partitions. The latter is a utility that builds hexahedral dominant grids starting from STL files. However, it is time and memory consuming and does not represent the optimal solution for gearboxes simulation. Another alternative is represented by cfMesh, an external grid generator that, in its base version, can be installed freely as a library that communicates with OpenFOAM ${ }^{\circledR}$. Even third-party software (commercial or opensource) can be exploited to create a mesh that can be converted into the OpenFOAM ${ }^{\circledR}$ format. One of the most used one is Salome [22], a powerful open-source pre-processor that can build polyhedral grids and has a Graphical User Interface (GUI) that makes the meshing process more intuitive. Since the correct setup of the CFD simulation of gearboxes is already demanding from a modeling point of view (aspects as multiphase conditions, dynamic mesh and turbulence must be considered simultaneously), it is fundamental to choose the meshing strategy that mostly reduces the complexity of the virtual model. In fact, the boundary rotation causes the distortion of the mesh which should be replaced with a new valid one at every little angular position. For this reason, a new algorithm called Global Remeshing Approach with Mesh Clustering $\left(\mathrm{GRA}^{\mathrm{MC}}\right)$ was implemented. This strategy is based on the creation of a limited number of numerical meshes that covers one engagement. Indeed, after the first engagement, the wheels find themselves in the exact same position as the one of the first mesh, thus meaning that the remeshing process can be reduced to the creation of predefined grids that, in turn, can be recursively reused to describe the whole rotation of the gears. By doing so, the amount of meshes needed is dramatically reduced to just few grids, leading to very high computational performance. Despite being simple considerations, the correct implementation of such algorithm requires a specific procedure based not only on the mesh quality indicators, but also on the design parameters of the gearbox. Moreover, all the libraries that control the time must be updated automatically at each mesh substitution. 
The first step is the creation of a high-quality mesh. In the authors experience, a maximum non-orthogonality of 70 and a maximum skewness of 2 are the threshold that should not be exceeded in these simulations. To find the number of meshes necessary to cover one engagement, a generic rotational speed is imposed to the gears. When the mesh quality exceeds the imposed limits, the simulation is stopped and the last valid timestep is taken as a reference $\left(t_{\text {up guess }}\right)$. The angle $(\alpha)$ between two successive teeth is calculated for each gear and the time needed for completing 1 engagement $\left(T_{\text {eng }}\right)$ can be obtained:

$$
\begin{gathered}
\alpha=\frac{2 \pi}{N_{\text {teeth }}} . \\
T_{\text {eng }}=\frac{\alpha}{\omega} .
\end{gathered}
$$

The following step is to identify the updating time of each mesh $\left(t_{u p}\right)$ that allows $T_{\text {eng }}$ to be reached with a set of predefined meshes. Exploiting the time $t_{\text {up guess }}$ previously found, the number of meshes needed to cover 1 engagement $\left(N_{\text {mesh }}\right)$ is then calculated as:

$$
N_{\text {mesh }}=\frac{T_{\text {eng }}}{t_{\text {upguess }}} \text {. }
$$

$N_{\text {mesh }}$ is then approximated to the nearest bigger integer number, and the $t_{\text {up guess }}$ is changed accordingly to the actual time $t_{u p}$ that will be used to update the grids through the engagement.

Once the mesh set with the wheels in predefined positions has been computed, it can be used for the entire simulation. The governing equations are solved subsequently for each grid, the results are saved and mapped onto the following mesh that is immediately provided as input. The results' interpolation follows a consistent strategy that guarantees that all variables are mapped between conformal domains. Furthermore, thanks to the very good control over the mesh generation parameters, the interpolation occurs between very similar grids in terms of elements' size and, therefore, the numerical errors are significantly reduced. At regime, the results are postprocessed. While the updating of the time libraries is performed at the end of each $t_{u p}$, the choice of which mesh to use at every step is managed by an if-else statement inside the procedure loop itself. The general workflow of the solution algorithm, which is completely automated in a Bash [23] script, is summarized in Fig. 1.

In the analyzed case, the final set of mesh is made of 10 grids. The time needed to create the set can be quantified in about 100 minutes. Without this algorithm, 230 mesh substitutions with a computational cost of 2,300 minutes would be necessary to cover just 1 gear complete rotation. Considering that for such simulation hundreds of mesh substitutions are necessary to reach convergence, the enormous advantages of the implemented algorithm can be clearly understood. The implementation of this strategy requires some coding to automatize the whole procedure.

\subsection{Numerical approach}

CFD codes are based on the solution of governing equations: mass, momentum, and energy conservation. In this study, the problem was modelled as isothermal. Therefore, the energy equation was not included in the calculation. In this way, the solution is limited to the mass and momentum equations which can be written as: 


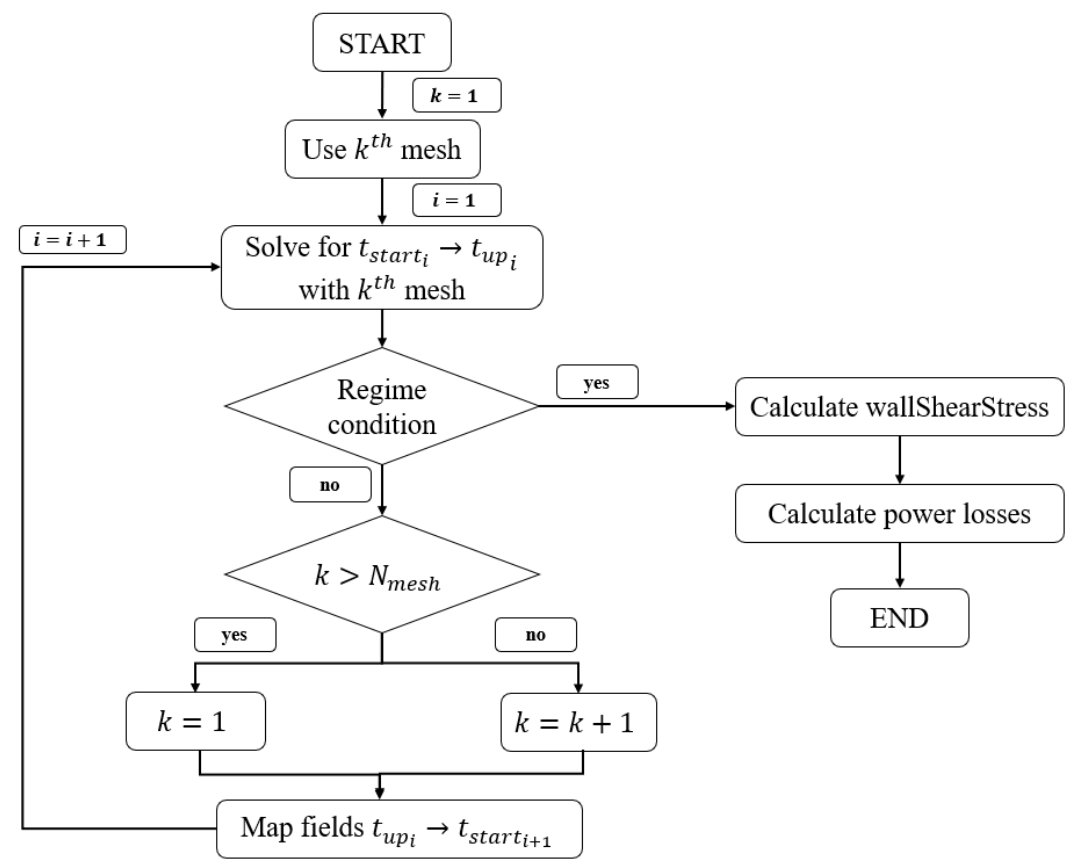

Figure 1: Scheme of the solution loop.

$$
\begin{gathered}
\frac{\partial \rho}{\partial t}+\nabla(\rho \boldsymbol{u})=0 . \\
\frac{\partial(\rho \boldsymbol{u})}{\partial t}+\nabla(\rho \boldsymbol{u} \boldsymbol{u})=-\nabla p+\nabla\left[\mu\left(\nabla \boldsymbol{u}+\nabla \boldsymbol{u}^{T}\right)\right]+\rho \boldsymbol{g}+\boldsymbol{F}
\end{gathered}
$$

These equations are valid only in simulations involving one phase. To model multiphase problems numerically, an additional balance equation to consider the presence of two or more phases must be added to the previous equation. By exploiting the volume of fluid (VOF) model [24], which is based on the definition of the scalar quantity volumetric fraction representing the percentage of one fluid in every cell of the domain, the multiphase problem can be solved. The equation of the volumetric fraction can be expressed as follows:

$$
\frac{\partial \alpha}{\partial t}+\nabla(\alpha \boldsymbol{u})=0
$$

The properties $\Theta$ of the different fluids (such as density and viscosity) are taken to define the properties of an equivalent fluid as follows:

$$
\Theta=\Theta \alpha+\Theta(1-\alpha)
$$

where $\Theta$ represents the generic property of each fluid.

The MULES (Multidimensional Universal Limiter with Explicit Solution) [25] correction can be added in the solver algorithm in order to obtain a more stable and bounded solution of the volumetric fraction field. This is accomplished by adding a dummy velocity field $\left(\boldsymbol{u}_{\boldsymbol{c}}\right)$ in the conservation equation of the volumetric fraction $\alpha$ : 


$$
\frac{\partial \alpha}{\partial t}+\nabla(\alpha \boldsymbol{u})+\nabla\left(\boldsymbol{u}_{\boldsymbol{c}} \alpha(1-\alpha)\right)=0
$$

An additional source term $\left(S_{g}\right)$ must be added to the equation to account for additional phenomena as cavitation:

$$
\left.\frac{\partial \alpha}{\partial t}+\nabla(\alpha \boldsymbol{u})+\nabla\left(\boldsymbol{u}_{c} \alpha(1-\alpha)\right)\right)=S_{g}
$$

To calculate the source term, a mathematical model must be introduced. The most used ones for describing cavitation are those by Kunz [26], Merkle et al. [27] and Saurer [28]. In this work, the Kunz model was used to model one operating condition (to account for cavitation, as will be explained in detail later). The great advantage of this formulation is related to the source term being independent of pressure. The vaporization $\left(\dot{m}_{v}\right)$ is modelled as proportional to the liquid fraction $(\alpha)$ and to the quantity of pressure under the saturation pressure $\left(p_{s a t}\right)$. The condensation $\left(\dot{m}_{c}\right)$ is modelled analogously:

$$
\begin{gathered}
\dot{m}_{v}=\frac{C_{v} \rho_{v}}{\frac{1}{2} \rho_{l} U_{\infty}^{2} t_{\infty}} \alpha \min \left[0, p-p_{\text {sat }}\right] . \\
\dot{m}_{c}=\frac{C_{c}}{\frac{1}{2} U_{\infty}^{2} t_{\infty}}(1-\alpha) \max \left[0, p-p_{s a t}\right] .
\end{gathered}
$$

\subsection{Analyzed system}

A helical gearbox is considered in the current analysis. The geometrical parameters are reported in Table 1 .

Table 1: Gears' geometrical characteristics.

\begin{tabular}{|l|c|c|}
\hline & Unit & Gears \\
\hline Transmission ratio & - & 1 \\
\hline Number of teeth & - & 23 \\
\hline Module & $\mathrm{mm}$ & 4 \\
\hline Helix angle & $\circ$ & 27 \\
\hline Face width & $\mathrm{mm}$ & 40 \\
\hline
\end{tabular}

A local size of $1 \mathrm{~mm}$ was imposed on the wheels' edges for refinement purposes, whereas a global size of $5 \mathrm{~mm}$ for the rest of the domain was used. A growth rate defining the difference between two adjacent elements of $20 \%$ was imposed. Five surface and volume optimization loops were utilized to improve the mesh quality indicators. These settings allowed one to obtain high quality meshes that even at the maximum deformation (just before the new valid mesh is provided) have a maximum non-orthogonality and a maximum skewness below 70 and 2, respectively. The total number of cells is about $520 \mathrm{k}$.

In Table 2 the simulated operating conditions are reported.

\subsection{Numerical schemes}

The PIMPLE (merged PISO-SIMPLE) algorithm was used in all simulations. This algorithm allows a better control in transient simulations. In fact, it is possible to tune the correctors of 
Table 2: Operating conditions.

\begin{tabular}{|l|c|c|c|}
\hline Operating condition & $\begin{array}{c}\text { Rotational speed } \\
(\mathrm{rpm})\end{array}$ & $\begin{array}{c}\text { Oil density } \\
\left(\mathrm{kg} / \mathrm{m}^{3}\right)\end{array}$ & $\begin{array}{c}\text { Oil viscosity } \\
\left(\mathrm{mm}^{2} / \mathrm{s}\right)\end{array}$ \\
\cline { 1 - 2 } Multiphase (centerline) & 3,000 & \multirow{3}{*}{800} & \multirow{2}{*}{0.001} \\
\cline { 2 - 2 } Complete filling & 6,000 & & \\
\cline { 2 - 2 } Complete filling with cavitation & 3,000 & & \\
\cline { 2 - 2 } & 6,000 & & \\
\cline { 2 - 2 } & 6,000 & & \\
\hline
\end{tabular}

the conservation equations within one timestep to reach the best compromise between computational effort and stability of the simulation. A convergence criterion of 1e -6 was imposed to all field's variables. The GAMG (Generalized Geometric-Algebraic Multi-Grid) solver was used for the pressure, while the velocity was solved with the Gauss-Seidel smooth solver. A maximum Courant number of 1 was imposed to ensure the stability of the simulations. The first-order implicit Euler scheme was used for time-stepping. A Total Variation Diminishing (TVD) scheme using the vanLeer limiter was adopted for the convection of the volumetric fraction.

\section{RESULTS}

Fig. 2 shows the lubricant distribution in the multiphase condition. The lubricant remains entrapped between the teeth and tends to move along it due to the helical configuration. Pressure peaks arise on the teeth flanks in contact with lubricant due to the higher inertial effects.
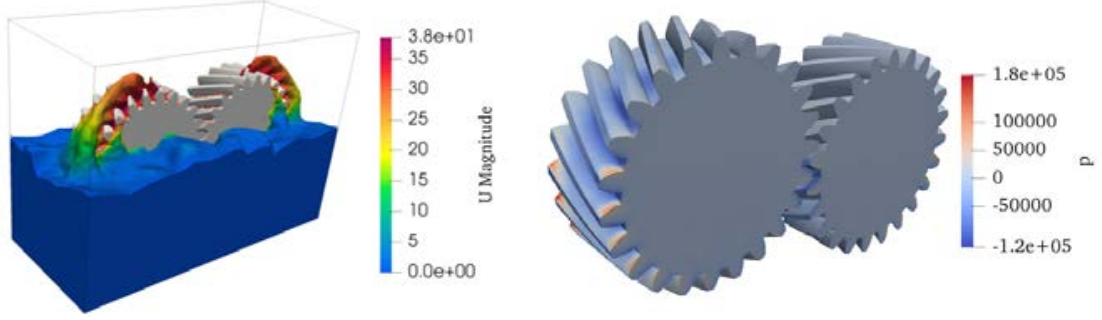

Figure 2: Lubricant distribution and pressure contour on the gears.

In Fig. 3 axial gradients in the meshing region are shown for the complete filled condition. These are related to the pressure increase in the meshing region. When the teeth leave the contact point, the pressure decreases, thus generating a suction effect which is highlighted by the axial velocity streamlines.

The simulation including cavitation shows that completely gearbox filling is not sufficient to ensure the complete wetting of the wheels. In fact, a pressurization is applied to accomplish this objective in practice [29]. If an external pressurization is not applied, local phase changes from liquid to vapour occur. In Fig. 4, the volumetric fraction field on a tooth flank is shown. Values lower than 1 indicate that the oil is cavitating. 

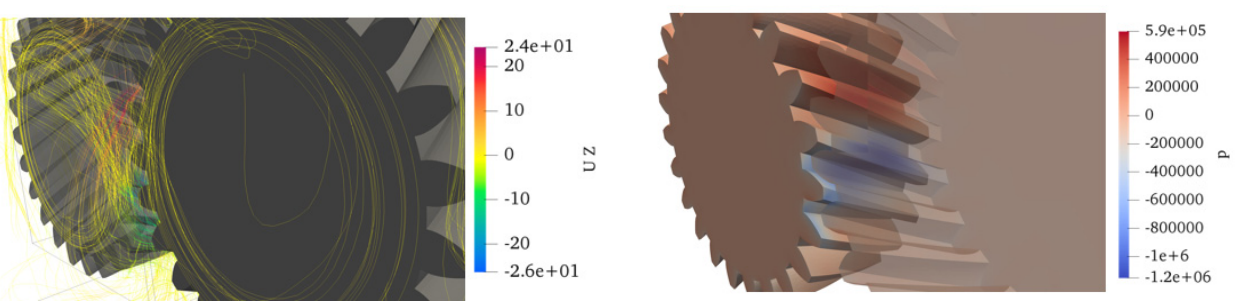

Figure 3: Axial velocity streamlines and pressure contour in the meshing zone.

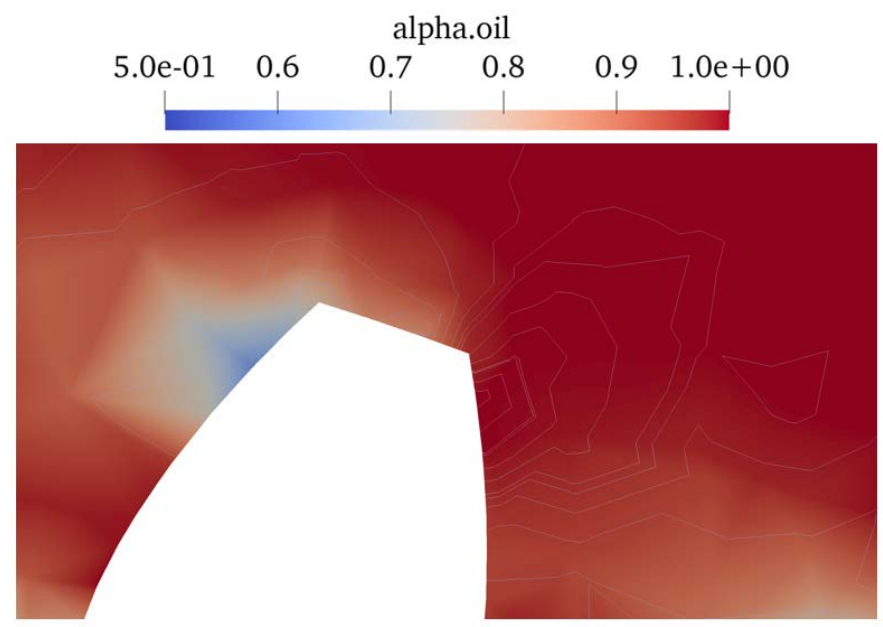

Figure 4: Lubricant contour on a slice of the gear in the cavitation case.

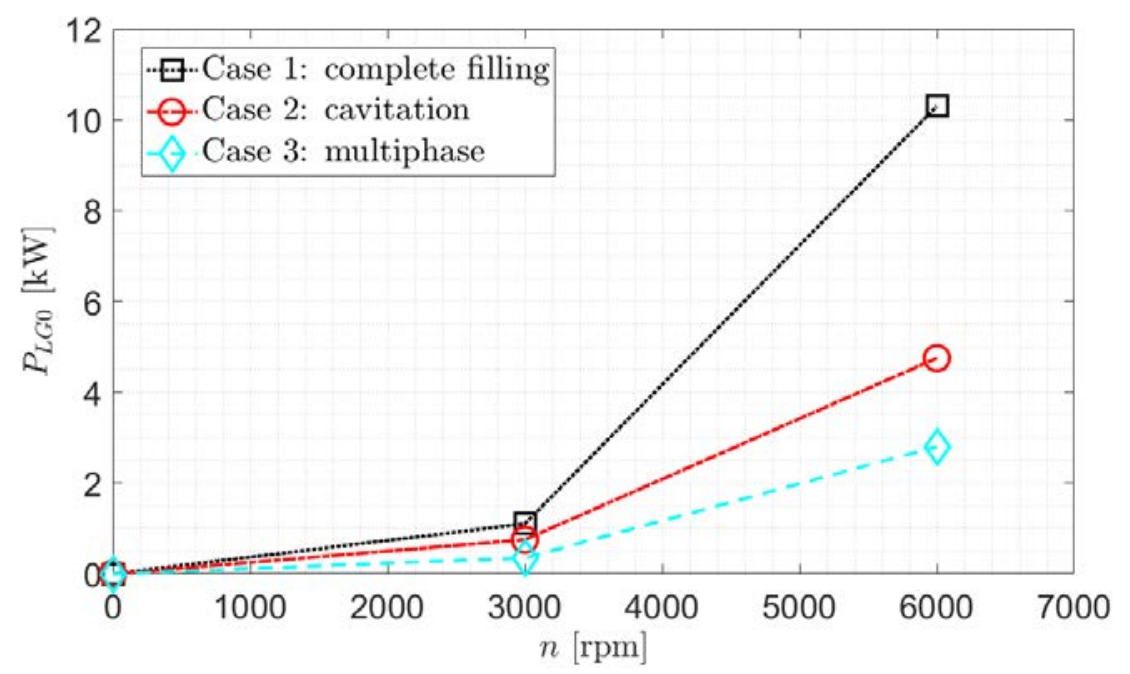

Figure 5: Power losses vs rotational speed for the different operating condition. 
The power dissipations for the three configurations is reported in Fig. 5. It can be noticed that the complete filling condition is characterized by the highest losses. This is mainly related to the windage effects, while in the multiphase condition is the oil splashing responsible for the power losses.

\section{COMPUTATIONAL PERFORMANCE}

The simulations were performed on a Deploy LXD Compute Node $\left(2 x I N T E L\right.$ Xeon ${ }^{\circledR}$ E2680, 8 Cores, $2.7 \mathrm{GHz}$ ). The simulations required on average $10 \mathrm{~h}$ each to reach convergence. The low computational time achieved parallelizing the computations among only 8 processors is mainly related to the implemented meshing algorithm that relies on the usage of just 10 meshes for the whole simulation. The computational resources dedicated to mesh management during the simulations are therefore drastically reduced and devoted just for the mapping process from mesh to mesh, since all the grids have been computed in advance. A comparison between the implemented GRA ${ }^{\mathrm{MC}}$ and a standard remeshing strategy that does not exploit the cyclicality of the gears' teeth position is reported in Table 3.

Table 3: GRA ${ }^{\mathrm{MC}}$ computational gain normalized to one gear rotation.

\begin{tabular}{|l|c|c|c|}
\hline Remeshing algorithm & STD approach & GRA $^{\mathrm{MC}}$ & Net gain $\%$ \\
\hline $\begin{array}{l}\text { Parametric value (1 rotation of the } \\
\text { wheel) }\end{array}$ & $t$ & $\frac{t}{N_{\text {teeth }}}$ & $\left(1-\frac{1}{N_{\text {teeth }}}\right) \times 100$ \\
\hline $\begin{array}{l}\text { Specific value for the analyzed } \\
\text { gears }\end{array}$ & $2,300 \mathrm{~min}$ & $100 \mathrm{~min}$ & $95.66 \%$ \\
\hline
\end{tabular}

Normalizing the computational effort to 1 gear rotation, the net gain associated with the explained mesh reduction algorithm can be quantified as $95.66 \%$. This has positive impact on the simulation time itself which is drastically reduced. All the time libraries are defined to ensure the wheels' passage in the control positions defined by the computed mesh set.

In Fig. 6, the relative time gain vs the number of gears rotation is shown for gears with different number of teeth. As the number of rotations increases, the effects of the GRA ${ }^{\mathrm{MC}}$

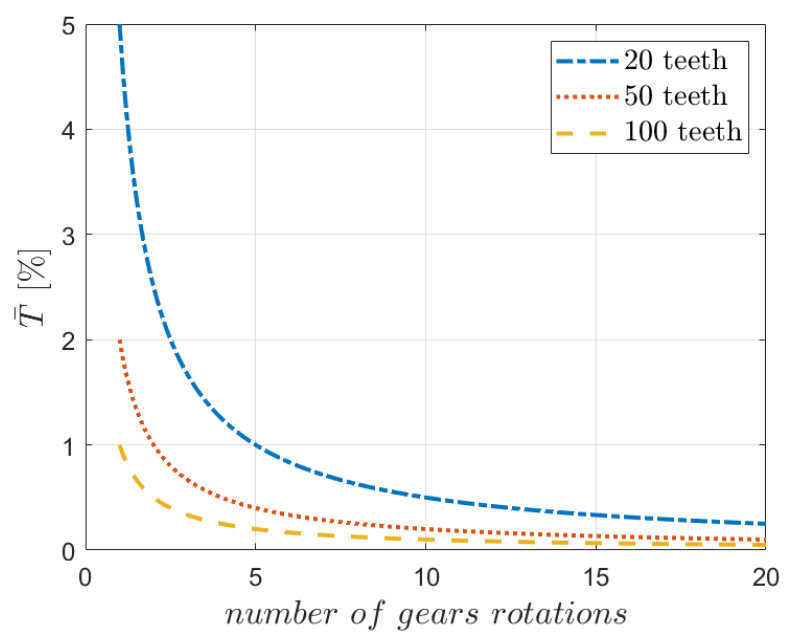

Figure 6: Meshing time for different gears' rotations and gears' size. 
become even more evident since the simulation time reduces continuously. This indicates that this algorithm represents an effective strategy to reduce the computational time in the CFD simulation of various gear types.

\section{CONCLUSIONS}

Differently from previous analyses, a complex domain as a helical gearbox was simulated in an opensource environment with a general procedure applicable to any configuration. The algorithm is based on the calculation of a set of grids that covers one engagement. This set is recursively reused at each engagement, thus limiting the complexity of the remeshing process. The effectiveness of the implemented procedure allowed a drastic reduction of the simulation time associated with the mesh management, which is one of the major concerns in these models. While the aim of this study was to show the capabilities of the new approach and the reduction of mesh requirements, from the simulations some interesting considerations emerged regarding the physics involved in lubricated systems and the relative differences in various operating conditions. Considering the complexity of obtaining such results experimentally, this methodology represents a time efficient solution even on a medium hardware without the need for big clusters.

\section{REFERENCES}

[1] Liu, H., Jurkschat, T., Lohner, T. \& Stahl, K., Determination of oil distribution and churning power loss of gearboxes by finite volume CFD method. Tribology International, 109, pp. 346-354, 2017. DOI: 10.1016/j.triboint.2016.12.042.

[2] Mastrone, M.N., Hartono, E.A., Chernoray, V. \& Concli, F., Oil distribution and churning losses of gearboxes: Experimental and numerical analysis. Tribology International, 151, 2020. DOI: 10.1016/j.triboint.2020.106496.

[3] Concli, F. et al., Load independent power losses of ordinary gears: Numerical and experimental analysis. 5th World Tribology Congress, WTC 2013, vol. 2, pp. 12431246, 2013. https://www.scopus.com/inward/record.uri?eid=2-s2.0-84919653123\& partnerID $=40 \&$ md5 $=1 \mathrm{df4c} 1 \mathrm{daa} 085 \mathrm{~b} 17 \mathrm{da} 52935814 \mathrm{f} 251 \mathrm{c} 5 \mathrm{c}$.

[4] Concli, F. \& Gorla, C., CFD simulation of power losses and lubricant flows in gearboxes. American Gear Manufacturers Association Fall Technical Meeting 2017, 2017.

[5] Concli, F. \& Gorla, C., Influence of lubricant temperature, lubricant level and rotational speed on the churning power loss in an industrial planetary speed reducer: Computational and experimental study. International Journal of Computional Methods \& Experimental Measurements, 1(4), pp. 353-366, 2013.

DOI: 10.2495/CMEM-V1-N4-353-366.

[6] Concli, F. \& Gorla, C., A CFD analysis of the oil squeezing power losses of a gear pair. International Journal of Computional Methods \& Experimental Measurements, 2(2), pp. 157-167, 2014. DOI: 10.2495/CMEM-V2-N2-157-167.

[7] Concli, F., Della Torre, A., Gorla, C. \& Montenegro, G., A new integrated approach for the prediction of the load independent power losses of gears: Development of a mesh-handling algorithm to reduce the CFD simulation time. Advances in Tribology, 2016, pp. 1-8, 2016. DOI: 10.1155/2016/2957151.

[8] Concli, F. \& Gorla, C., Influence of lubricant temperature, lubricant level and rotational speed on the churning power loss in an industrial planetary speed reducer: Computational and experimental study. International Journal of Computional Methods \& Experimental Measurements, 1(4), pp. 353-366, 2013. 
[9] Burberi, E., Fondelli, T., Andreini, A., Facchini, B. \& Cipolla, L., CFD simulations of a meshing gear pair. Proceedings of the ASME Turbo Expo, vol. 5A-2016, 2016.

DOI: $10.1115 /$ GT2016-57454.

[10] Concli, F., Low-loss gears precision planetary gearboxes: Reduction of the load dependent power losses and efficiency estimation through a hybrid analyticalnumerical optimization tool [Hochleistungs- und Präzisions-Planetengetriebe: Effizienzschätzung und Reduzierun]. Forschung im Ingenieurwesen/Engineering Research, 81(4), pp. 395-407, 2017. DOI: 10.1007/s10010-017-0242-0.

[11] Bianchini, C., Da Soghe, R., Errico, J.D. \& Tarchi, L., Computational analysis of windage losses in an epicyclic gear train. Proceedings of the ASME Turbo Expo, vol. 5B-2017, 2017. DOI: 10.1115/GT2017-64917.

[12] Dai, Y., Ma, F., Zhu, X., Su, Q. \& Hu, X., Evaluation and optimization of the oil jet lubrication performance for orthogonal face gear drive: Modelling, simulation and experimental validation. Energies, 12(10), 2019. DOI: 10.3390/en12101935.

[13] Ferrari, C. \& Marani, P., Study of air inclusion in lubrication system of CVT gearbox transmission with biphasic CFD simulation. BATH/ASME 2016 Symposium on Fluid Power \& Motion Control, 2016. DOI: 10.1115/FPMC2016-1767.

[14] Hu, X., Jiang, Y., Luo, C., Feng, L. \& Dai, Y., Churning power losses of a gearbox with spiral bevel geared transmission. Tribology International, 129, pp. 398-406, 2019. DOI: 10.1016/j.triboint.2018.08.041.

[15] Peng, Q., Zhou, C., Gui, L. \& Fan, Z., Investigation of the lubrication system in a vehicle axle: Numerical model and experimental validation. Proceedings of the IMechE Part D: Journal of Automobile Engineering, 233(5), pp. 1232-1244, 2019. DOI: $10.1177 / 0954407018766128$.

[16] Peng, Q., Gui, L. \& Fan, Z., Numerical and experimental investigation of splashing oil flow in a hypoid gearbox. Engineering Applications of Computational Fluid Mechanics, 12(1), pp. 324-333, 2018. DOI: 10.1080/19942060.2018.1432506.

[17] Dai, Y., Jia, J., Ouyang, B. \& Bian, J., Determination of an optimal oil jet nozzle layout for helical gear lubrication: Mathematical modeling, numerical simulation, and experimental validation. Complexity, 2020, 2020. DOI: 10.1155/2020/2187027.

[18] Deng, X. et al., Lubrication mechanism in gearbox of high-speed railway trains. Journal of Advanced Mechanical Design, Systems \& Manufacturing, 14(4), 2020. DOI: 10.1299/jamdsm.2020jamdsm0054.

[19] Deng, X., Wang, S., Hammi, Y., Qian, L. \& Liu, Y., A combined experimental and computational study of lubrication mechanism of high precision reducer adopting a worm gear drive with complicated space surface contact. Tribology International, 146, 2020. DOI: 10.1016/j.triboint.2020.106261.

[20] Morhard, B., Schweigert, D., Mileti, M., Sedlmair, M., Lohner, T. \& Stahl, K., Efficient lubrication of a high-speed electromechanical powertrain with holistic thermal management. Forschung im Ingenieurwesen/Engineering Research, 2020. DOI: $10.1007 / \mathrm{s} 10010-020-00423-0$.

[21] OpenFOAM. http://www.openfoam.com.

[22] Salome. http://www.salome-platform.org.

[23] Bash. www.gnu.org/software/bash.

[24] Hirt, C.W. \& Nichols, B.D., Volume of fluid (VOF) method for the dynamics of free boundaries. Journal of Computional Physics, 39(1), pp. 201-225, 1981.

[25] Rusche, H., Computational Fluid Dynamics of Dispersed Two-Phase Flows at High Phase Fractions, Imperial College of Science, Technology and Medicine: London, 2002. 
[26] Kunz, R.F. et al., Preconditioned Navier-Stokes method for two-phase flows with application to cavitation prediction. Computers \& Fluids, 29(8), pp. 849-875, 2000.

[27] Merkle, C.L., Feng, J. \& Buelow, P.E.O., Computational modeling of the dynamics of sheet cavitation. 3rd International Symposium on Cavitation, pp. 47-54, 1998.

[28] Saurer, J., Instationären kaviterende Sträömung - Ein neues Modell, basierend auf Front Capturing (VoF) and Blasendynamik, Universität Karlsruhe, 2000.

[29] Höhn, B.R., Michaelis, K. \& Otto, H.P., Influences on no-load gear losses. 3rd European Conference on Tribology, pp. 639-644, 2011. 